\title{
Externally provoked freezing of gait in open runways in advanced Parkinson's disease results from motor and mental collapse
}

\author{
C. Moreau $\cdot$ L. Defebvre $\cdot$ S. Bleuse $\cdot$ \\ J. L. Blatt $\cdot$ A. Duhamel $\cdot$ B. R. Bloem . \\ A. Destée $\cdot$ P. Krystkowiak
}

Received: 25 January 2008/ Accepted: 20 July 2008/Published online: 23 August 2008

(c) The Author(s) 2008. This article is published with open access at Springerlink.com

\begin{abstract}
Freezing of gait (FOG) in Parkinson's disease (PD) is defined as a sudden inability to maintain effective stepping movements. However, its pathophysiology remains unclear. The objectives are: (1) To assess the contribution of both spatial (walking speed, stride length) and temporal parameters (cadence, stride time) and their coefficients of variation to the genesis of FOG in PD. (2) To evaluate whether and how externally imposed modifications of self-determined gait would elicit FOG. We included ten patients with advanced $\mathrm{PD}$, and with daily off drug FOG episodes. We focused on walking in an open runway. For each subject, we manipulated gait by externally imposing four changes in walking speed and four changes in cadence. FOG episodes, often with a long duration of more than 5 -s, were observed mostly under
\end{abstract}

C. Moreau · L. Defebvre · A. Destée · P. Krystkowiak

Department of Neurology, EA2683, IFR 114, Lille, France

e-mail: c-moreau@chru-lille.fr

S. Bleuse - J. L. Blatt

Department of Clinical Neurophysiology,

EA2683, IFR 114, Lille, France

A. Duhamel

CERIM, Chru Lille, Lille, France

B. R. Bloem

Department of Neurology, Fonders Center for Neuroscience

Radboud, University Nijmegen Medical Center,

Nijmegen, The Netherlands

L. Defebvre $(\bowtie)$

Clinique Neurologique, Hôpital R. Salengro, CHU,

59037 Lille Cedex, France

e-mail: ldefebvre@chru-lille.fr conditions with a high imposed cadence. The steps that immediately preceded these episodes were mainly characterized by an increase in cadence and an increase in stride length variability. The results also underscore that FOG can be elicited in a laboratory setting when patients are placed under considerable strain, at least in advanced stages of PD. Patients were unable to adequately negotiate the extreme imposed cadence condition, and this resulted in frequent FOG episodes, even while walking in an open runway. Placing advanced PD patients into extreme imposed conditions leads to a motor wise and mental collapse response, culminating in FOG. Future work should establish the relevance of these findings for the more common forms of FOG, including brief episodes during turning or gait initiation.

Keywords Freezing of gait · Parkinson's disease ·

Variability · Hypokinesia

\section{Introduction}

Gait disturbances are common in Parkinson's disease (PD). Decreased arm swing is one of the first signs, followed by reduced speed and stride length (Morris et al. 1996). Freezing of gait (FOG) is an incapacitating gait disorder that typically appears in advanced stages of PD, although FOG may occasionally be present in patients with early and untreated PD (Nieuwboer et al. 2001; Bloem et al. 2004). FOG is operationally defined clinically as a sudden inability to voluntarily maintain effective stepping movements during gait (Giladi 2006) FOG mainly occurs during gait initiation (in $85 \%$ of patients), when turning (45\%) or when walking through a narrow passage or doorway $(25 \%)$ (Bloem et al. 2004). 
Gait disorders observed in early stages of PD are characterized by a reduced stride length, which is a primary determinant of hypokinesia, and this worsens as the disease progresses (Morris et al. 1994a, b, 1996, 1998). This difficulty in regulating stride length is present even though patients can still modulate cadence under varying conditions. The observed higher cadence in PD (seen for any given imposed velocity) might reflect a compensatory mechanism (Morris et al. 1994a, b, 1998).

No data are available concerning the evolution of these abnormalities in very advanced stages of the disease; however, after 10 years of evolution, freezing commonly leads to falls and may result in loss of independence, without any effective treatments (Bloem et al. 2004).

Biomechanical analysis of gait prior to a freezing episode showed an exponential decrease in stride length with a concomitant increase in cadence (Nieuwboer et al. 2001). Rhythmicity, as defined by stride time regularity (the inverse of cadence), seems to be specifically affected in basal ganglia diseases (PD and Huntington's disease) (Haussdorf et al. 1998). Stride time variability (as defined by its coefficient of variation, CV) is the only gait parameter which increases markedly in PD patients with FOG, even in between overt episodes of FOG (Haussdorf et al. 2003). Increased variability could thus be a marker for the primary locomotor arhythmicity which ultimately leads to FOG (Haussdorf et al. 2003). However, all these spatial parameters (velocity, stride length) and temporal parameters (cadence, stride time) were never studied simultaneously in PD patients presenting with FOG.

Manipulating the walking speed or cadence may be another way to study FOG. Indeed, PD patients have lost the ability to flexibly adjust their walking pattern according to the actual demands of the situation at hand (Morris et al. 1994a, b, 1998, ). In daily life or even under experimental conditions, patients may well select a walking speed or cadence that is least prone to provoke FOG, as they are used to do in everyday life. Several groups have taken this to the test, but with contradictory results. One study manipulated gait cadence in patients with advanced PD patients and FOG, and observed that the greatest number of FOG episodes occurred at the highest frequencies (Turnbull et al. 1996). However, in another study the highest CV for stride time (as a surrogate marker for FOG) (Haussdorf et al. 2003) was observed for an imposed cadence $30 \%$ below the subject's spontaneous rate (Ebersbach et al. 1999). These findings underscore the need for a single study where the impact of imposed variations in cadence and velocities on FOG is studied within the same cohort of patients.

Therefore, the aim of our present study was to analyse spontaneous gait as well as the impact of four imposed changes in velocity and four imposed changes in cadence conditions in advanced PD patients with FOG. To resolve some of the issues outlined above, we simultaneously measured all of the spatial parameters (velocity, stride length) and temporal parameters (cadence, stride time). In order to specify the kinematic abnormalities which could lead to a FOG episode, we specifically analysed the five strides before an overt FOG episode.

\section{Patients and methods}

\section{Patients}

Ten subjects with idiopathic PD (as defined by the UK Brain Bank criteria) were recruited from the Outpatient Clinic of the Movement Disorders Unit. In order to select subjects with FOG, we delivered the "Freezing of Gait Questionnaire" (Ebersbach et al. 1999) to 120 PD patients. Based on the answers, we selected ten patients with daily and disabling FOG episodes (during the off drug phase). Subjects were excluded if they were unable to walk alone without assistance in the off drug condition (Hoehn and Yahr stage $\geq 4$ ), if they had significant co-morbidity that was likely to affect gait, or if patients were demented (we excluded patients with a Mattis scale score < 130).

We determined the unified Parkinson's disease rating scale (UPDRS) motor score for each patient in the practically defined off drug condition (i.e., following withdrawal of all antiparkinson medication for at least $12 \mathrm{~h}$ ). All gait analyses were also done in the off drug condition. The study was approved by the local investigational review board and each patient signed an informed consent form prior to testing.

\section{Gait protocol}

Patients were instructed to walk without physical support along a 7-m walkway. A six-camera, optoelectronic system (VICON 370 from Oxford Metrics, Oxford, UK, sampling frequency $50 \mathrm{~Hz}$ ) was used to determine gait kinematics (Giladi et al. 2000).

Our specific test procedure consisted of three parts. At the start of day 1, patients were asked to walk normally at a comfortable speed (i.e., under spontaneous conditions). Five trials were performed and then analyzed in order to calculate the mean spontaneous gait velocity $(\mathrm{m} / \mathrm{s})$ and cadence (strides/min) for at least ten strides.

In the second part of day 1 (about $1 \mathrm{~h}$ after the baseline condition), subjects were asked to follow a mobile target mounted on a rail attached to the ceiling. Four different target velocities (calculated relative to each individual patient's mean, spontaneous velocity) were imposed. The imposed velocities were set to 20 and $40 \%$ below (Velocity Minus: VM40, VM20) and 20 and $40 \%$ above (Velocity 
Plus: VP20, VP40) the individual's spontaneous velocity. Twenty and $40 \%$ were calculated according to normal range of age matched subjects for normal and high gait speeds in the Laboratory, in order to specify the strategy that PD patient would adopt and effects on FOG.

In the third part of the experiment (performed on day 2), patients were instructed to adapt their stepping rate to the rhythm set by a metronome. The four imposed frequencies were calculated relative to each individual patient's mean spontaneous cadence. The imposed cadences values were set to 20 and $40 \%$ below (Cadence Minus, CM40, CM20) and above (Cadence Plus, CP20 and CP40) the individual's spontaneous cadence.

Five trials were recorded for each imposed velocity or cadence. The subject was not informed in advance of which condition was under investigation and was merely instructed to follow the target or the rhythm. The order of each of these conditions was altered randomly between subjects.

\section{Observation-based definition of freezing events}

The "gold standard" was the occurrence of clinically overt FOG episodes, as defined by independent clinical inspection of the patient by two neurologists with extensive experience in movement disorders. FOG was only accepted when both raters independently agreed that FOG was present. FOG episodes were defined by a sudden and involuntary cessation of gait ("block") (Bloem et al. 2004; Ebersbach et al. 1999). In order to include "certain" FOG, we only included episodes lasting at least $5 \mathrm{~s}$. However, separate analyses of brief FOG episodes (which may be more common in daily life) revealed very similar results (data not shown).

\section{Data analysis}

Spatial and temporal kinematic parameters (including velocity $(\mathrm{m} / \mathrm{s})$, cadence (stride/min), stride length $(\mathrm{m})$, stride time (s)) were calculated using the Workstation and Polygon $^{\odot}$ software packages from Oxford Metrics. We analyzed outside FOG strides for spontaneous gait and each imposed gait condition, as well as the five strides preceding each FOG episode under imposed conditions.

First, for each kinematic parameter, the mean value for each imposed cadence or velocity condition was compared with the mean for the spontaneous condition. Second, our chosen index of stride-to-stride variability was the coefficient of variation for each kinematic parameter $(\mathrm{CV}=100 \times$ standard deviation/mean $)$. We compared $\mathrm{CV}$ s in spontaneous versus imposed cadence and velocity conditions for the following parameters: cadence, velocity, stride length and stride time. Finally, for the imposed condition with the highest incidence of FOG episodes, the kinematic parameters (median and CV) for the five strides preceding an episode were compared with the values recorded for five other consecutive strides (i.e., far from a FOG episode) under the same condition.

\section{Statistical analysis}

We performed Wilcoxon non-parametric tests for each of the comparisons. A Bonferroni post hoc test was additionally used for correction. A statistical significance level of 0.01 was chosen.

\section{Clinical characteristics}

Median age of the 10 patients was 70 years [first quartile (Q1) 68; third quartile (Q3) 70.2] and median disease duration was 18 years [13;20]. The median UPDRS III score in the off drug condition was 40 [34; 44.5].

One hundred twenty patients' average score at the FOG questionnaire was $24( \pm 11)$ (Ebersbach et al. 1999). The average "FOG subscore" (including items 4, 5, 6, 7) was $7.5( \pm 4)$.

\section{Results}

Number of freezing episodes (Table 1)

All patients presented FOG in the highest imposed cadence condition (CP40). Secondly, a high number of FOG episodes was also observed in the highest velocity condition (VP40) in 6 patients.

Gait modulation during the imposed conditions (Table 2)

Patients were able to modulate their gait cadence as instructed. We observed a statistically significant difference when comparing the medians for each imposed

Table 1 Numbers of FOG episodes and FOG suffers in each of the eight imposed conditions

\begin{tabular}{lllllllll}
\hline & VM 40 & VM 20 & VP 20 & VP 40 & CM 40 & CM 20 & CP 20 & CP 40 \\
\hline Number of FOG episodes observed & 3 & 12 & 6 & 15 & 4 & 1 & 6 \\
Number of patients (out of 10) with FOG & 2 & 6 & 2 & 6 & 3 & 1 & 26 \\
\hline
\end{tabular}


Table 2 Median values of kinetic parameters for each condition

\begin{tabular}{|c|c|c|c|c|c|c|c|c|c|}
\hline & \multirow[t]{2}{*}{ SP } & \multicolumn{4}{|c|}{ Imposed cadence conditions } & \multicolumn{4}{|c|}{ Imposed velocity conditions } \\
\hline & & CM 40 & CM 20 & CP 20 & CP 40 & VM 40 & VM 20 & VP 20 & VP 40 \\
\hline Cadence (s/min) & 55 & $36^{*}$ & $44^{*}$ & $62 *$ & $68^{*}$ & 52 & 58 & 61 & $65^{*}$ \\
\hline Velocity $(\mathrm{m} / \mathrm{s})$ & 0.6 & $0.3^{*}$ & $0.45^{*}$ & 0.6 & 0.6 & $0.39 * *$ & 0.56 & 0.66 & $0.82 *$ \\
\hline Stride length (m) & 0.72 & $0.58^{*}$ & $0.61 *$ & $0.54 *$ & $0.57 * *$ & $0.36 * *$ & $0.6^{*}$ & $0.64 *$ & 0.74 \\
\hline Stride time (s) & 1.1 & $1.7 *$ & $1.45^{*}$ & 1 & 0.9 & 1.1 & 1 & $0.98^{*}$ & $0.91 *$ \\
\hline
\end{tabular}

Cadence (strides/min); velocity $(\mathrm{m} / \mathrm{s})$; stride length $(\mathrm{m})$ and stride time $(\mathrm{s})$

* Statistical significance: Wilcoxon and Bonferroni post hoc: ${ }^{*} P<0.01 ; * * P<0.001$

cadence and the median for spontaneous cadence (Table 2). Cadences as executed during the imposed conditions were fairly homogeneous and comparable to the imposed values.

In contrast, patients found it more difficult to adjust their walking velocity as instructed. Thus, walking velocity only differed from spontaneous gait for the VM40 and VP40 conditions. Moreover, for all patients, executed velocities were lower than the imposed ones under most experimental conditions.

A significant decrease in stride length was observed when the imposed cadence was high (CP20 and CP40 conditions), but patients were unable to increase their stride length when the imposed velocity was high (VP20 and VP40 conditions), with a paradoxical and significant stride length decrease for the VP20 condition. In the VP40 condition, the median executed velocity was lower than the imposed velocity, due to an insufficient increase in stride length - a mechanism not compensated for by the significant increase in cadence. A significant and parallel reduction in stride time with increasing cadence was observed for high imposed velocity conditions (VP20 and VP40).

\section{Gait variability (Table 3)}

For all kinematic parameters, variability was significantly higher under the slowest imposed cadence and velocity conditions (CM40 and VM40). No significant increase in the CVs for cadence and stride time was observed for the conditions with the highest number of FOG episodes (CP40 and VP40).

Analysis of strides preceding FOG (Table 4)

When comparing the five strides immediately before a FOG episode in the CP40 condition with a series of five other strides (i.e., well outside a FOG episode) under the same imposed condition, we found a significant increase in the mean cadence before a FOG episode, with reductions in stride length, stride time and velocity. No significant variations in the $\mathrm{CV}$ values for cadence or stride time were observed before a FOG episode, whereas significant increases in stride length variability and velocity variability were seen.

\section{Discussion}

The main findings of this study are as follows. First, imposing variations in spontaneous gait in patients with advanced PD can provoke clear FOG episodes. Specifically, a high cadence and, to a lesser extent, an imposed higher walking velocity can induce FOG. These FOG episodes were fairly lengthy (often more than 5-s duration), and occurred during walking in an open runway. Second, this occurrence of overt FOG episodes permitted us to perform a detailed kinematic analysis of spatial and

Table 3 Median values of coefficients of variations (CV) of kinematics parameters for each condition

\begin{tabular}{|c|c|c|c|c|c|c|c|c|c|}
\hline \multirow{2}{*}{$\begin{array}{l}\mathrm{CV} \\
(\%)\end{array}$} & \multirow[t]{2}{*}{ SP } & \multicolumn{4}{|c|}{ Imposed cadence conditions } & \multicolumn{4}{|c|}{ Imposed velocity conditions } \\
\hline & & CM 40 & CM 20 & CP 20 & $\mathrm{CP} 40$ & VM 40 & VM 20 & VP 20 & VP 40 \\
\hline CV Cadence & 5.2 & $9,5^{*}$ & $6^{*}$ & 7 & 7 & $13 * *$ & 8 & $9 *$ & 6 \\
\hline CV Velocity & 7.3 & $17 * *$ & $12 * *$ & 9 & 8 & $20 * *$ & 11 & 11 & 9,5 \\
\hline CV Stride length & 6.8 & $13 * *$ & 12 & 11 & 12 & $17 * *$ & $14^{* *}$ & 11 & 11 \\
\hline CV Stride time & 5.3 & $12 * *$ & 6 & 7 & 5 & $12 * *$ & $8^{*}$ & 10 & 6 \\
\hline
\end{tabular}

$\mathrm{CV}$ of cadence (strides/min); velocity $(\mathrm{m} / \mathrm{s})$; stride length $(\mathrm{m})$ and stride time $(\mathrm{s})$

* Statistical significance: Wilcoxon and Bonferroni post hoc: $* P<0.01 ; * * P<0.001$ 
Table 4 Comparison of the median values for the 5 strides preceding a FOG episode in the CP 40 imposed condition versus 5 other consecutive strides (i.e., far from FOG episodes) in the same condition

\begin{tabular}{lllll}
\hline CP 40 condition & Cadence & Velocity & Stride length & Stride time \\
\hline 5 strides before a FOG episode & $88^{*}$ & $0.36^{*}$ & $0.22^{*}$ & $0.72^{*}$ \\
CV & 9.8 & $21.2^{\dagger}$ & $26.5^{\dagger}$ & 10.3 \\
5 FOG free strides & $70^{*}$ & $0.51^{*}$ & $0.45^{*}$ & $0.88^{*}$ \\
CV & 7.8 & $12.9^{\dagger}$ & $14.8^{\dagger}$ & 8.68 \\
\hline
\end{tabular}

Statistical significance for values: $* P<0.01$

$\mathrm{CV}$ : variability coefficient: ${ }^{\dagger} P<0.01$

temporal gait parameters that immediately preceded a clear FOG episode. These episodes were mainly characterized by an increase in cadence and stride length variability.

FOG is notoriously difficult to evoke under experimental conditions, perhaps because the anxiety associated with being tested can suppress FOG (a form of kinesia paradoxa). It was therefore interesting to see that an externally imposed and fairly drastic change in self-chosen gait parameters commonly led to FOG in our subjects. Indeed, a high cadence (up to $40 \%$ above the naturally chosen value) was associated with prolonged ( $>5 \mathrm{~s}$ duration) FOG episodes in all of our subjects. Unnaturally high walking velocities also provoked FOG, albeit in only six of our patients. However, we should point out, firstly, that our patient population was fairly selected (advanced PD), so the present data cannot be automatically generalized to the overall PD population. Indeed, our patients had advanced and longstanding disease (average disease duration was 18 years), and this clearly exceeds the mean disease duration of about 12 years in most recent FOG studies (Giladi 2006; Davis et al. 2006). However, most of our kinematic values are comparable with those of previous studies (Haussdorf et al. 1998, 2003).

Secondly, all of our present patients had significant FOG in daily life, with prolonged off drug FOG episodes even in open runways, as reflected by their scores on the FOG questionnaire. We should note that it remains to be seen whether FOG can also be elicited in more mildly affected patients with less prominent FOG in everyday life. On the other hand, we focused on gait in an open runway, where FOG rarely occurs in daily life. FOG most commonly occurs while turning or upon gait initiation, and we suspect that under such circumstances, FOG may also be seen in milder patients when the gait task is rendered more demanding, as was done in the present study.

Concerning our analysis of kinematic parameters, previous data of gait in spontaneous versus imposed conditions showed that advanced PD patients are still able to modulate gait cadence (Krystkowiak et al. 2003). In the present study, cadences rates were still correctly modulated for almost all of the conditions (Table 2). The ability to modulate walking velocity was less well preserved in the advanced PD patients. Walking velocities that were actually executed during testing were lower than the imposed ones under most experimental conditions, and the most extreme conditions merely induced an increase in cadence in our PD patients, whereas no modulation of stride length was possible, despite the external cue (a moving target) that was provided.

Moreover, the mean CVs observed for each of the four gait parameters during spontaneous walking were about twice those seen in control subjects, and this is in agreement with previously reported data (Haussdorf et al. 2003). Maximal CV values were observed for the lowest imposed velocity and cadence conditions (CM40 and VM40). No correlation was found between the $\mathrm{CV}$ values and the incidence of FOG episodes (Haussdorf et al. 1998, 2003). The stride-to-stride gait variability seems to be either a byproduct of bradykinesia (correlated with lower gait speed) or an intrinsic perturbation of the basal ganglia (not specifically related to FOG in PD) (Frenckel-Toledo et al. 2005). The same pattern emerges from our analysis of the five strides preceding a FOG episode. A significant increase in cadence (accompanied by a decrease in velocity, stride length and stride time) was observed 3 steps before a FOG episode, as previously described (Nieuwboer et al. 2001). However, in contrast to the report from Nieuwboer et al. we did not find any increase in the CV for stride time or cadence, but a significant increase in stride length variability. Finally, we cannot exclude that a specific interhemispheric timing problem (i.e., a deficit in finetuning the temporal gait parameters of both legs in relation to each other) also contributes to FOG (Plotnik et al. 2005, 2007).

Another important point to discuss is the way our patients were able to mentally handle these extreme and unnatural imposed situations. Stressful dual task condition could elicit FOG episodes (Yogev Seligmann et al. 2008), especially in PD patients with executive dysfunction (Amboni et al. 2008). After 18 years of disease duration, most of the patients presented executive dysfunction (with deficits in attention) and slight cognitive impairment. The neuropsychological influences on walking are manifested by the individual awareness of a destination, the ability to 
appropriately control the limb movements that produce gait, and the ability to navigate within often complex environs to successfully reach the desired goal (Yogev Seligmann et al. 2008). Executive dysfunction combined to an impaired limb control could have elicited FOG in extremely high cadences conditions.

Subjects were asked to focus on their gait and to concentrate on imposed velocities or cadences, materialized by a visual target or an auditory cue; the time of processing these "dual task" increased in our patients because of their limited capacities to share attention. Like in the neuropsychological Bottleneck theory, our patients were unable to treat the control of gait and the attention to external cues (Yogev Seligmann et al. 2008). As described for the posture, PD patients choose the "gait second strategy", and FOG became the outcome of this mental and motor collapse (Bloem et al. 2006).

\section{Conclusion}

In light of these results, we hypothesize that in our patients, FOG corresponds to the extreme end of the hypokinesia spectrum but is less strongly related to stride time variability (which would suggest that a primary neural timing deficit underlies FOG) (Giladi 2006).

Putting these patients into extreme imposed conditions leads to a motor wise and mental collapse response, as reflected by fairly prolonged off state FOG that occurs even during walking in an open runway. Future work should establish the relevance of these findings for the more common forms of FOG, including brief episodes during turning or gait initiation.

Acknowledgment Dr. Bastiaan R. Bloem was supported by a ZonMw VIDI grant (\#016.076.352).

Open Access This article is distributed under the terms of the Creative Commons Attribution Noncommercial License which permits any noncommercial use, distribution, and reproduction in any medium, provided the original author(s) and source are credited.

\section{References}

Amboni M, Cozzolino A, Longo K et al (2008) Freezing of gait and executive functions in patients with Parkinson's disease. Mov Dis 23:395-400
Bloem BR, Haussdorf J, Visser J, Giladi N (2004) Fall and freezing of gait in PD: a review of two interconnected, episodic phenomena. Mov Dis 19(8):871-884

Bloem B, Grimbergen YA, van Dijk JG et al (2006) The posture second strategy: a review of wrong priorities in Prakinson's disease. J Neurol Sci 248:196-204

Davis J, Lyons K, Pahwa R (2006) Freezing of gait after bilateral subthalamic nucleus stimulation for Parkinson's Diseaese. Clin Neurol Neurosurg 108(5):461-464

Ebersbach G, Heijmenberg M, Kinderman L et al (1999) Interference of rhythmic constraint on gait in healthy subjects and patients with early PD: evidence of impaired locomotor pattern generation in early PD. Mov Dis 4:619-625

Frenckel-Toledo S, Giladi N, Peretz C, Herman T et al (2005) Effects of gait speed on gait rhythmicity in Parkinson 's disease: variability of stride time and swing time respond differently. J NeuroEng Rehab 2:23

Giladi N (2006) Oral presentation, 10th edn. Movement disorders Society, Kyoto

Giladi N, Shabtaï H, Simon ES et al (2000) Construction of freezing of gait questionnaire for patients with Parkinsonism. Parkinsonism Relat Disord 6:165-170

Haussdorf J, Cudkowicz M, Firtion R et al (1998) Gait variability and basal ganglia disorders: stride-to-stride variations of gait cycle timing in PD and HD. MovDis 13:428-437

Haussdorf J, Schaafsma JD, Balash Y et al (2003) Impaired regulation of stride variability in PD subjects with freezing of gait. Exp Brain Res 149:187-194

Krystkowiak P, Blatt JL, Bourriez JL, Duhamel A et al (2003) Effects of subthalamic nucleus stimulation and levodopa treatement on gait abnormalities in Parkinson's disease. Arch Neurol 60:80-84

Morris M, Iansek R, Matyas T, Summers J (1994a) The pathogenesis of gait hypokinesia in PD. Brain 117:1169-1181

Morris M, Iansek R, Matyas T et al (1994b) Ability to modulate walking cadence remains intact in Parkinson's disease. JNNP 57:1532-1534

Morris M, Iansek R, Matyas T et al (1996) Stride length regulation in Parkinson's disease. Brain 119:551-568

Morris ME, Iansek R, Matyas TA, Summers J (1998) Abnormalities in the stride length cadence relation in parkinsonian gait. Mov Dis 13:61-69

Nieuwboer A, Dom R, De Weert W et al (2001) Abnormalities of the spatio temporal characteristics of gait at the onset of freezing in PD. Mov Dis 16:1066-1075

Plotnik M, Giladi N, Balash Y, Peretz C, Hausdorf JM (2005) Is freezing of gait in Parkinson's disease related to asymmetric motor function? Ann Neurol 57:656-663

Plotnik M, Giladi N, Hausdorff JM (2007) A new measure for quantifying the bilateral coordination of human gait: effects of aging and Parkinson's disease. Exp Brain Res 181:561-570

Turnbull G, Lund K, Millican L, Perry K, et al. (1996) The effect of different frequencies of auditive cueing oon the temporal and spatial gait parameters of people with Parkinsonism. Phys Canada 48 (2): abstract 3

Yogev Seligmann G, Haussdorf J, Giladi N (2008) The role of executive function and attention in gait. Mov Dis 23:329-342 